\title{
Generalized Ratio-Cum-Product Estimator for Estimating Population Mean in Systematic Sampling.
}

\author{
Rafia Jan ${ }^{1 *}$, Asra Nazir ${ }^{2}$,Showkat Maqbool ${ }^{3}$ and T.R. Jan ${ }^{4}$ \\ ${ }^{1,2,4}$ Department of Statistics, University of Kashmir, J\&K, India \\ ${ }^{3}$ Division of Agricultural Statistics and Economics, FOA, Wadura, Skuast, Kashmir, India
}

\section{Available online at: www.isroset.org}

Accepted 12/Aug/2018, Online 30/Aug/2018

\begin{abstract}
This manuscript suggests a generalized class of ratio-cum-product estimator in systematic sampling. The Mean Square Error (MSE) of the suggested class and mathematical conditions have been also derived to prove the efficiency of proposed estimators. The results are justified numerically.
\end{abstract}

Keywords Auxiliary variate; Bias; Efficiency; Mean Squared Error.

\section{INTRODUCTION}

Systematic sampling was studied by [1]. In literature, many authors have discussed the estimation procedures in systematic sampling for instance [2], [3], [4], [5], [6], [7], [8] and [9].

\section{NOTATIONS AND SOME EXISTING ESTIMATORS}

Let $y_{i j}$ and $x_{i j}$ denote the variate $y$ and the variate $x$ respectively bearing the serial number $i+(j-1) k$ in the population $(i=1,2, \ldots ., k ; j=1,2, \ldots ., n)$. If the $i^{\text {th }}$ sampling unit is randomly taken from the first $k$ units, then $\bar{y}_{s y s}$ and $\bar{x}_{s y s}$ are defined as

$\bar{y}_{s y s}=\bar{y}_{i .}=\frac{1}{n} \sum_{j=1}^{n} y_{i j}, \bar{x}_{s y s}=\bar{x}_{i .}=\frac{1}{n} \sum_{j=1}^{n} x_{i j}$.

[10] proposed the ratio estimator in systematic sampling given by

$$
\bar{y}_{R}^{s y s}=\bar{y}_{s y s}\left(\frac{\bar{X}}{\bar{x}_{s y s}}\right),
$$

And MSE of the estimator is given as

$$
\operatorname{MSE}\left(\bar{y}_{R}^{\text {sys }}\right)=\lambda \bar{Y}^{2}\left(\rho_{y}^{*} C_{y}^{2}+\rho_{x}^{*} C_{x}^{2}\left(1-2 K \sqrt{\rho^{* * *}}\right)\right),
$$

where

$$
\begin{aligned}
& K=\rho_{x y} \frac{C_{y}}{C_{x}}, \rho_{y}^{*}=\left\{1+\rho_{y}(n-1)\right\}, \rho_{x}^{*}=\left\{1+\rho_{x}(n-1)\right\}, \\
& \rho^{* *}=\left(\frac{\rho_{y}^{*}}{\rho_{x}^{*}}\right), \rho_{y x}=\frac{S_{y x}}{S_{y} S_{x}}, \lambda=\frac{N-1}{n N} \\
& S_{y x}=\frac{1}{N-1} \sum_{i=1}^{k} \sum_{j=1}^{n}\left(x_{i j}-\bar{X}\right)\left(y_{i j}-\bar{Y}\right), \\
& S_{y}^{2}=\frac{1}{N-1} \sum_{i=1}^{k} \sum_{j=1}^{n}\left(y_{i j}-\bar{Y}\right)^{2}, S_{x}^{2}=\frac{1}{N-1} \sum_{i=1}^{k} \sum_{j=1}^{n}\left(x_{i j}-\bar{X}\right)^{2} .
\end{aligned}
$$

The product estimator in systematic sampling given by [11] is

$$
\bar{y}_{P}^{s y s}=\bar{y}_{s y s}\left(\frac{\bar{x}_{s y s}}{\bar{X}}\right),
$$

And the expression (MSE) is given as

$$
\operatorname{MSE}\left(\bar{y}_{P}^{\text {sys }}\right)=\lambda \bar{Y}^{2}\left(\rho_{y}^{*} C_{y}^{2}+\rho_{x}^{*} C_{x}^{2}\left(1+2 K \sqrt{\rho^{* *}}\right)\right) .
$$

[12] suggested the exponential ratio and exponential product estimator in systematic sampling given as

$$
\bar{y}_{E R}^{s y s}=\bar{y}_{s y s} \exp \left(\frac{\bar{X}-\bar{x}_{s y s}}{\bar{X}+\bar{x}_{s y s}}\right) \text {, }
$$




$$
\bar{y}_{E P}^{s y s}=\bar{y}_{s y s} \exp \left(\frac{\bar{x}_{s y s}-\bar{X}}{\bar{x}_{s y s}+\bar{X}}\right) .
$$

The MSE of the exponential ratio and exponential product estimator are given as

$$
\operatorname{MSE}\left(\bar{y}_{E R}^{s y s}\right)=\lambda \bar{Y}^{2}\left(\rho_{y}^{*} C_{y}^{2}+\rho_{x}^{*}\left(\frac{C_{x}^{2}}{4}\right)\left(1-4 K \sqrt{\rho^{* * *}}\right)\right)
$$

$\operatorname{MSE}\left(\bar{y}_{E P}^{s y s}\right)=\lambda \bar{Y}^{2}\left(\rho_{y}^{*} C_{y}^{2}+\rho_{x}^{*}\left(\frac{C_{x}^{2}}{4}\right)\left(1+4 K \sqrt{\rho^{* *}}\right)\right)$

\section{PROPOSED ESTIMATOR}

Following [13] we define a class of the ratio-cum-product estimators in systematic sampling given as

$T=\bar{y}_{s y s}\left[\frac{a \bar{X}+b}{a \bar{x}_{s y s}+b}\right]^{g}\left[\frac{a \bar{x}_{s y s}+b}{a \bar{X}+b}\right]^{1-g}$

$=\bar{y}_{s y s}\left[\frac{a \bar{X}+b}{a \bar{x}_{s y s}+b}\right]^{2 g-1}$,

where $g$ is a suitable chosen scaler.

For obtaining the bias and MSE, we write $\bar{y}=\bar{Y}\left(1+e_{0}\right), \bar{x}=\bar{X}\left(1+e_{1}\right)$,

such that

$$
E\left(e_{0}\right)=E\left(e_{1}\right)=0
$$

and

$$
\begin{aligned}
& E\left(e_{0}{ }^{2}\right)=\lambda \cdot C_{y}{ }^{2} \rho_{y}^{*}, E\left(e_{1}{ }^{2}\right)=\lambda \cdot C_{x}{ }^{2} \rho_{x}^{*}, \\
& E\left(e_{0} e_{1}\right)=\lambda \cdot K C_{x}^{2} \sqrt{\rho_{y}^{*} \rho_{x}^{*}} .
\end{aligned}
$$

Expressing equation (9) in terms of $e^{\prime} s$, we get

$$
T=\bar{Y}\left(1+e_{0}\right)\left(1+\theta e_{1}\right)^{-(2 g-1)},
$$

where $\theta=\frac{a \bar{X}}{(a \bar{X}+b)}$.

Solving equation (10) and retaining the terms up to second order, we have

$$
(T-\bar{Y})=\bar{Y}\left(\begin{array}{l}
e_{0}-(2 g-1) \theta e_{0} \\
-(2 g-1) \theta e_{0} \theta e_{1}+g(2 g-1) \theta^{2} e_{1}^{2}
\end{array}\right) .
$$

Squaring above equation both sides, we have

$$
(T-\bar{Y})^{2}=\bar{Y}^{2}\left[e_{0}^{2}-2(2 g-1) \theta e_{0} e_{1}+(2 g-1)^{2} \theta^{2} e_{1}^{2}\right]
$$

The MSE is given as

$$
\operatorname{MSE}(T)=\lambda \bar{Y}^{2}\left[\begin{array}{c}
C_{y}^{2} \rho_{y}^{*}+\theta^{2} C_{x}^{2} \rho_{x}^{*}(2 g-1)^{2} \\
-2 \theta K C_{x}^{2} \sqrt{\rho_{y}^{*} \rho_{x}^{*}}(2 g-1)
\end{array}\right] .
$$

We differentiate the $\operatorname{MSE}(T)$ with respect to $g$ and equating the derivative to zero, $g_{\text {opt }}$ thus obtained is given by,

$$
g_{o p t}=\frac{1}{2}\left[1+\frac{K \sqrt{\rho_{y}^{*} \rho_{x}^{*}}}{\theta \rho_{x}^{*}}\right]=\frac{1}{2}\left[1+\frac{K \sqrt{\rho^{* * *}}}{\theta}\right],
$$

using the value of $g_{o p t}$, we get the $M S E_{\text {min }}(T)$ given as

$$
M S E_{\min }(T)=\gamma \bar{Y}^{2} \rho_{y}^{*} C_{y}^{2}\left(1-\rho_{x y}^{2}\right) .
$$

\section{EFFICIENCY COMPARISONS}

We have derived the conditions under which the proposed estimator is better than the usual unbiased estimator, ratio estimator, product estimator is given as under

Table 1: Conditions for Comparison

\begin{tabular}{|l|l|}
\hline $\begin{array}{l}\text { Existing } \\
\text { estimator }\end{array}$ & Conditions \\
\hline $\bar{y}_{s y s}$ & $\lambda \bar{Y}^{2} \rho_{y}^{*} C_{y}^{2} \rho_{x y}^{2}>0$ \\
\hline $\bar{y}_{R}^{\text {sys }}$ & $\lambda \bar{Y}^{2} \rho_{x}^{*} C_{x}^{2}\left(1-K \sqrt{\rho^{* *}}\right)>0$ \\
\hline $\bar{y}_{P}^{s y s}$ & $\lambda \bar{Y}^{2} \rho_{x}^{*} C_{x}^{2}\left(1+K \sqrt{\rho^{* *}}\right)>0$ \\
\hline
\end{tabular}




\section{Numerical Illustrations}

In order to show that our estimator is more efficient numerically, we have used the data given by [14].

Table 2: Some Special Cases of the Proposed Class.

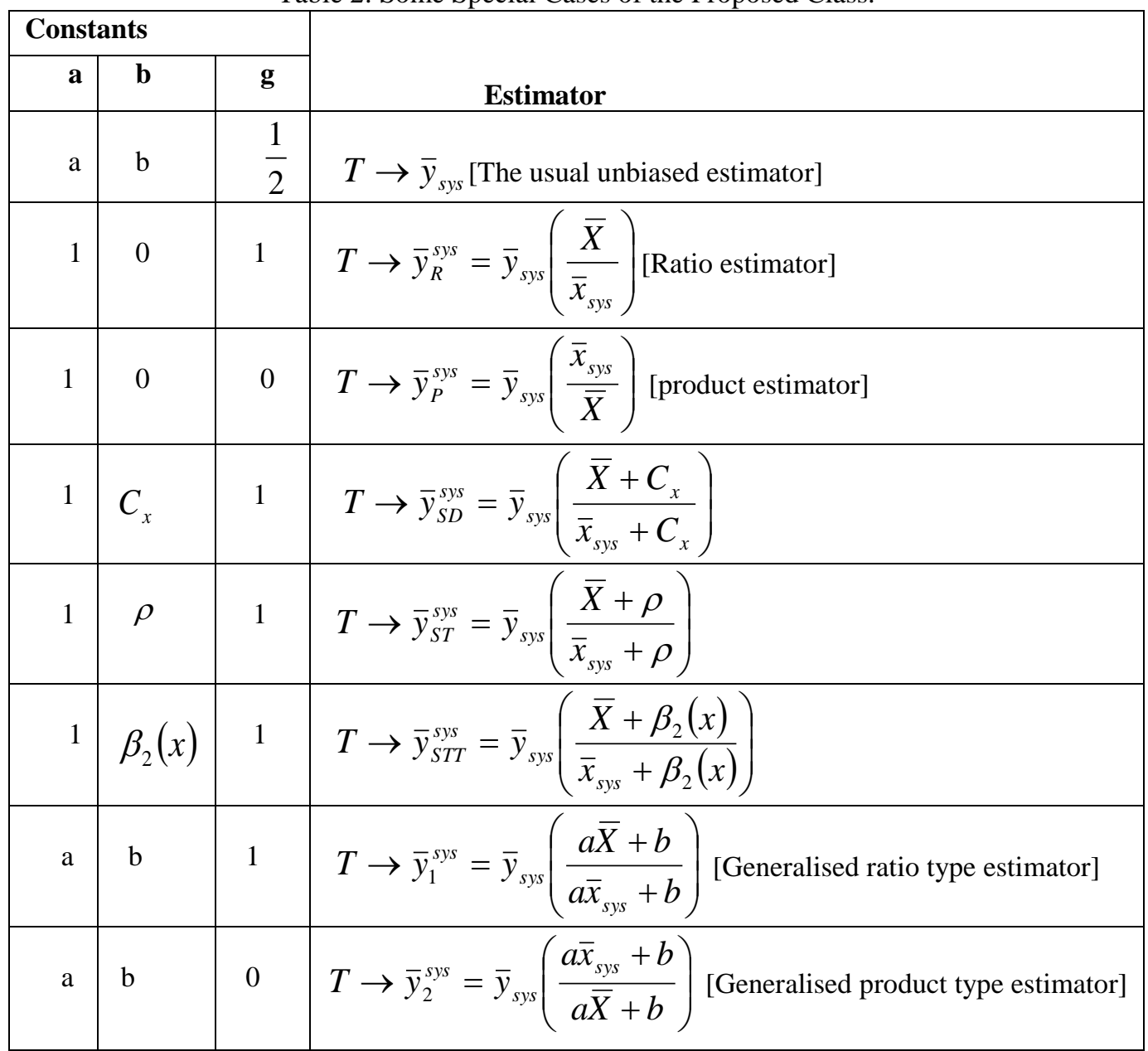

Table 3: Data Statistics

\begin{tabular}{|l|l|l|l|}
\hline$N$ & 15 & $C_{y}$ & 0.56 \\
\hline$n$ & 3 & $S_{x}^{2}$ & 149.55 \\
\hline $\bar{Y}$ & 80 & $C_{x}$ & 0.28 \\
\hline $\bar{X}$ & 44.47 & $S_{x y}$ & 538.57 \\
\hline$\rho_{x y}$ & 0.9848 & $\rho_{x}$ & 0.707 \\
\hline$S_{y}^{2}$ & 2000 & $\rho_{y}$ & 0.6652 \\
\hline
\end{tabular}

Table 4 shows the (MSE) and Percent Relative Efficiency (PRE) of various estimators given as,

\begin{tabular}{|l|l|l|}
\hline Estimators & MSE & PRE \\
\hline $\bar{y}_{\text {sys }}$ & 1455.08 & 100.00 \\
\hline $\bar{y}_{R}^{\text {sys }}$ & 373.32 & 398.62 \\
\hline $\bar{y}_{P}^{\text {sys }}$ & 786.06 & 189.45 \\
\hline $\bar{y}_{E R}^{\text {sys }}$ & 820.09 & 177.43 \\
\hline & & 139.42 \\
\hline $\bar{y}_{E P} y s$ & 1044.42 & 3196.57 \\
\hline$T$ & 45.74 & \\
\hline
\end{tabular}




\section{CONCLUSION}

We suggested a generalised ratio-cum-product estimator in systematic sampling and its properties are derived. We have also evaluated the performance of the estimator by using the real life data set. From table 3 it is observed that the estimator is better than the existing estimators, thus it is preferable to use in practical surveys.

\section{REFERENCES}

[1] W. G. Madow and L. H. Madow, "On the theory of systematic sampling”, Ann. Math. Statist., Vol. 15, pp. 1-24, 1944.

[2] D. B. Lahiri, "On the question of bias of systematic sampling", Proceedings of World Population Conference, 6, pp. 349-362, 1954.

[3] W, Gautschi, "Some remarks on systematic sampling", Ann. Math. Statist.,Vol. 28, pp. 385-394, 1957.

[4] J. Hajeck, "Optimum strategy and other problems in probability sampling", Casopis pro Pestovani Matematiky, Vol. 84, pp. 387-423, 1959.

[5] W. G. Cochran, "Relative efficiency of systematic and stratified random samples for a certain class of population", Ann. Math. Statist., Vol. 17, pp. 164-177, 1946.
[6] K. S. Kushwaha and H. P. Singh, "Class of almost unbiased ratio and product estimators in systematic sampling" Jour. Ind. Soc. Ag. Statistics, Vol. 41 Issue 2, pp. 193-205, 1989.

[7] Banarasi, S. N. S. Kushwaha, and K. S. Kushwaha, "A class of ratio, product and difference $(R P D)$ estimators in systematic sampling", Microelectron. Reliab., Vol. 33 Issue 4, pp. 455457, 1993.

[8] R. Singh, and H. P. Singh "Almost unbiased ratio and product type estimators in systematic sampling", Questiio, Vol. 22 Issue 3, pp. 403-416, 1998.

[9] R. Singh, S. Malik, and V. K. Singh. "An improved estimator in systematic sampling”, Jour. of Scie. Res., Vol. 56, pp. 177-182, 2012.

[10] A. K. P. C. Swain, "The use of systematic sampling ratio estimate" J. Ind. Statist. Assoc., Vol. 2, pp.160-164, 1964.

[11] N. D. Shukla, "Systematic sampling and product method of estimation", In Proceedings of all India Seminar on Demography and Statistics. B.H.U. Varanasi: India, 1971.

[12] H. P. Singh, and N. K. Jatwa, "A class of exponential type estimators in systematic sampling”, Eco. Qulty. Control., Vol. 27, pp. 195-208, 2016.

[13] H. P. Singh, R, S. Solanki and A. K. Singh, "A generalized ratio-cum-product estimator for estimating the finite population mean in survey sampling", Commun. Stat. Theory Methods Vol. 45 Issue 1, pp. 158-172, 2016.

[14] R. Tailor, N. K. Jatwa and H. P. Singh, "A ratio-cum-product estimator of finite population mean in systematic sampling", Statistics in Transition, Vol. 14, Issue 3, pp. 391-398, 2013. 